\title{
Linked-up loops: a reliable means of control
}

Depending on how you look at it, large gene-expression networks are dauntingly complicated or, if you're a mathematical modeller, elegantly simple and reducible to a discrete number of meaningful ON/OFF switches. A modelling study has begun to link these network 'building blocks' together by showing that an optimal output is obtained when two such switches are combined.

There are several well-characterized classes of switch-like elements, but this study concentrated on positive-feedback loops, in which $A$ activates $B$ and $B$ activates $A$. Feedback loops operate in many signalling pathways, with some processes - such as oocyte maturation in frogs and the polarization of yeast cells - relying on more complicated switch arrangements, such as the coupling of two positive-feedback loops. This arrangement is initially rather puzzling: why combine two switches when a single one will carry out exactly the same function?
A computational modelling approach revealed a reason for the extra layer of complexity: slow loops are stable switches, but cannot transit between states quickly; by contrast, fast loops make for unstable (noisy) switches, but are quick at switching between states. Coupling two switches of the same kind together brings no overall benefit over having a single switch, but combining a fast and a slow switch yields an optimal output - that of a fast yet robust, reliable switch.

This type of dual positive-feedback loop is the one that is seen in the sub-circuits of many biological systems, and we now understand why this might be. This work also highlights the comforting thought that the details of a circuit are in fact dispensable, provided we have a good handle on the wiring.

\section{Tanita Casci}

(4) References and links

ORIGINAL RESEARCH PAPER

Brandman, O. et al. Interlinked fast and slow positive feedback loops drive reliable cell divisions. Science 310, 496-498 (2005)

FURTHER READING Bornholdt, S. Less is more in modelling large genetic networks. Science $\mathbf{3 1 0}$, 449-451 (2005) | Wall, M. E. et al. Design of gene circuits: lessons from bacteria. Nature Rev. Genet. 5, 34-42 (2004)

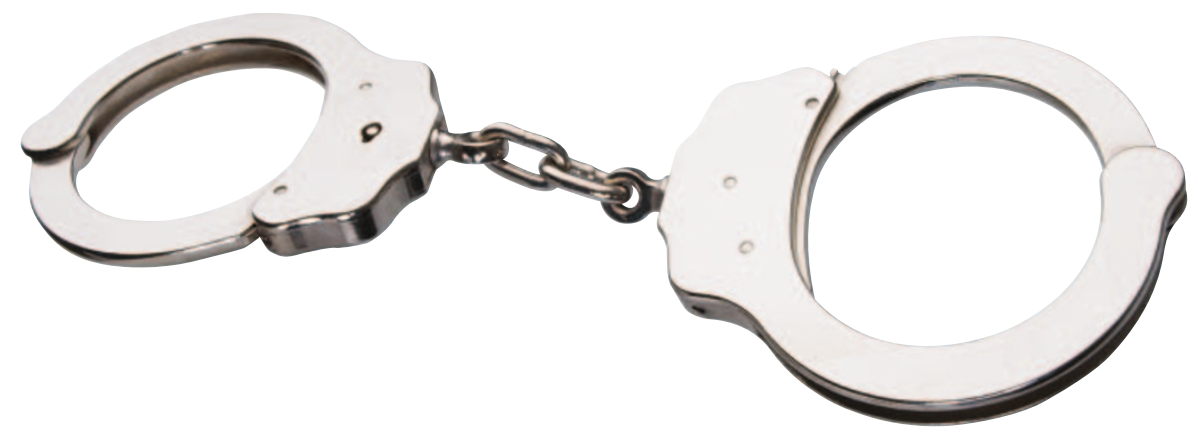

